\title{
Androgens in Women - Critical Evaluation of the Methods for Their Determination in Diagnostics of Endocrine Disorders
}

\author{
M. DUŠKOVÁ ${ }^{1,2}$, L. KOLÁTOROVÁ ${ }^{1}$, L. STÁRKA ${ }^{1}$ \\ ${ }^{1}$ Department of Steroids and Proteofactors, Institute of Endocrinology, Prague, Czech Republic, \\ ${ }^{2}$ Department of Medicine Strahov, General University Hospital, Prague, Czech Republic
}

Received June 12, 2018

Accepted June 27, 2018

\begin{abstract}
Summary
The androgens dehydroepiandrosterone sulfate, dehydroepiandrosterone, androstenedione and testosterone are routinely assessed in women, and circulating levels of these androgens reflect their production. These androgens are measured in most laboratories using various immuno-analytical methods. Recently, however, androgen assays have begun to be performed using gas or liquid chromatography combined with mass spectrometry. To better understand the difficulties and issues of androgen laboratory diagnostics, it is important to assess each of the methods used, how and why they were introduced into practice, and their advantages, limits, historic milestones and current status. It is also necessary to understand how reference ranges are determined and specifics arising from the physiology of individual androgens. Here we present a summary and discussion of these issues.
\end{abstract}

\section{Key words}

Direct immunoassay • LC-MS • Testosterone • Androstenedione • $\operatorname{DHEA}(\mathrm{S}) \bullet$ RIA with extraction

\section{Corresponding author}

M. Dušková, Institute of Endocrinology, Národní 8, 11694 Prague 1, Czech Republic. E-mail: mduskova@endo.cz

\section{Introduction}

Four androgens are routinely measured in women: dehydroepiandrosterone sulfate, dehydroepiandrosterone, androstenedione, and testosterone. In general, circulating levels of these androgens reflect their production. They are primarily measured in serum or plasma, and this seems to be most appropriate, but they can also be measured in mucus and urine. The advantages and disadvantages of each body fluid are still the subject of much discussion.

Increased androgenization in women can be caused by either a higher level of androgens - termed hyperandrogenemia - or a higher sensitivity of target organs to androgens, the levels of which may actually be within reference ranges. It must be noted that women are much more sensitive to androgens than men. Changes in the levels of testosterone that lead to hirsutism in women cause no changes in hair richness in men. Moderate androgenization in women is manifested in dermatological symptoms such as acne, hirsutism or hair loss, while more severe forms can lead to somatic changes such as an enlarged clitoris, a deepening of the voice, and changes to body structure. Such severe forms can also be seen accompanying ovarian or adrenal gland tumors that produce androgens, or in non-treated congenital adrenal hyperplasia $(\mathrm{CAH})$. A less severe form of androgenization is typical for more common female endocrine disorders such as polycystic ovarian syndrome and in minor or treated forms of $\mathrm{CAH}$, as well as in so-called idiopathic hirsutism.

The diagnosis of hyperandrogenemia in women is accompanied by many difficulties. These difficulties can be divided into several types, and each is critical for correct interpretations of results. The fundamentals are a correct indication for examination, the choice of analytes to be measured, following the correct procedures in the preanalytic phase that must take physiological changes in steroid hormones into account, the possible 
effects of medications used or dietary supplements, and the overall health of the patient.

Another complicating issue is the method used to measure androgens in the laboratory. For the actual measurements and choice of method, considerations of accuracy, cost, and automatization all play a role. These considerations will be different for routine diagnostic examinations and research purposes. Laboratory assays are also complicated by the issue of free androgens. In the blood, about $65 \%$ of testosterone circulates bound to sex hormone binding globulin (SHBG), about 30-34 \% bound to albumin, and only $2-3 \%$ as free testosterone. Total testosterone is the most basic diagnostic parameter, but other forms of testosterone may sometimes importantly contribute to evaluating the status of the patient. Another difficulty with laboratory assays of androgens is the problem of reference ranges, which should not just be automatically taken from other references but should be tested and confirmed in each laboratory. Reference ranges in women should also take into account the phase of the menstrual cycle and the age factor. Below, we provide a detailed description of the individual aspects of laboratory assays of androgens that should be mastered in order to correctly interpret results.

\section{Individual androgens - physiology and function}

\section{Dehydroepiandrosterone sulfate (DHEAS)}

DHEAS is quantitatively the most abundant circulating steroid hormone and reaches high micromolar concentrations in the blood, several orders of magnitude higher than other steroid hormones that typically have nanomolar concentrations. These high concentrations can be measured directly using radioimmunoassay (RIA), since possible interference from other steroids is marginal.

Concentrations of DHEAS change with age. They begin to increase at adrenarche, peak between 20 and 30 years of age, and then start to decline. At 70 years old, women have only about $20 \%$ of the levels they had at 20 years. DHEAS levels do not markedly change during the day or during the menstrual cycle. It is produced in the adrenal gland, so changes throughout the day might be expected, but because of the high concentrations slight daily changes are typically not seen. In one of our studies (Stárka et al. 2015) we managed to find some minor changes depending on time of day and diet, but such changes should present no obstacle to interpretations of results for samples taken at non-standard times. Levels of DHEAS in fertile, pregnant and postmenopausal women in plasma are shown in Table 1.

DHEAS is produced directly as a sulfate in the adrenal glands at a daily rate of 3.5-20 mg. It has a minor influence on many metabolic processes and is active as a neurosteroid. It is itself just slightly androgenic, but can serve as a precursor to androgens and estrogen in the periphery. It is generally not useful in diagnosing hyperandrogenemia except in cases of extremely high levels accompanying some adrenal tumors, primarily cancers, or as a signal of the onset of adrenarche in girls.

\section{Dehydroepiandrosterone (DHEA)}

DHEA is measured in some laboratories using extraction methods, while in others the less-optimal method of direct immunoassay is used.

Changes in DHEA concentrations over the course of life are similar to DHEAS. However, in contrast to DHEAS DHEA has a clear diurnal profile, matching that of cortisol (Stárka et al. 2015). Changes during the menstrual cycle are minor, with a slight increase in the late luteal phase (Šrámková et al. 2015). Levels of DHEA in fertile, pregnant and postmenopausal women in plasma are shown in Table 1.

DHEA is produced at much lower concentrations than DHEAS, totaling around 3-8 $\mu \mathrm{g}$. About half is produced in the adrenal glands, and the other half either secreted by the ovaries or converted from the sulfate in the periphery. Concentrations in the circulatory system range between 3-35 nmol/1, depending strongly on age. In conclusion measurements of nonconjugated DHEA are not useful in diagnosing hyperandrogenemia.

\section{Androstenedione}

Androstenedione is measured in some laboratories using extraction methods, while in others the less-optimal method of direct immunoassay is used.

The daily production of $1.4-6.2 \mathrm{mg}$ in women is divided equally between the ovaries and the adrenal glands. Androstenedione changes during the menstrual cycle similarly to testosterone, increasing during the periovulation period by about $25 \%$. It also has a circadian rhythm. A slight drop in androstenedione with age has been described. Fertile women have higher androstenedione levels than postmenopausal women (Stanczyk 2006). Smokers have higher androstendione 
levels than non-smokers throughout their lives (Dušková et al. 2012, Jandíková et al. 2014, Adamcová et al. 2017). Levels of androstendione in fertile, pregnant and postmenopausal women in plasma are shown in Table 1.

Androstenedione does not have significant androgenic activity, but it is a direct precursor of testosterone. For this reason it is useful to measure when diagnosing hyperandrogenism.

\section{Testosterone}

Testosterone is primarily measured using the RIA method, with the disadvantages mentioned below. It is recommended that extraction should be used when measuring testosterone in women, but while some laboratories do offer extraction methods, direct RIA without extraction is still the most widely used method in Europe (Pugeat 2017).
Concentrations of testosterone change during the menstrual cycle similarly as androstenedione, with a periovulatory increase of about $25 \%$ caused by increased production in the ovaries (Šrámková et al. 2015). Descriptions in the literature of changes during the day are inconsistent; a study in our laboratory found only insignificant slight changes (Rácz et al. 2015). In women, testosterone is produced in the ovaries and adrenal glands, from which follow changes both during the menstrual cycle as well as during the day. Several authors have described lowered testosterone levels with age. Smokers have higher testosterone levels than non-smokers throughout their lives (Dušková et al. 2012, Jandíková et al. 2014, Adamcová et al. 2017). Levels of testosterone in fertile, pregnant and postmenopausal women in plasma are shown in Table 1.

Table 1. Levels of DHEA, DHEAS, androstendione and testosterone in fertile, pregnant and postmenopausal women in plasma.

\begin{tabular}{|c|c|c|c|c|c|}
\hline & $\begin{array}{c}\text { DHEA } \\
(\mathrm{nmol} / \mathrm{l})\end{array}$ & $\begin{array}{l}\text { DHEAS } \\
(\mu \mathrm{mol} / \mathrm{l})\end{array}$ & $\begin{array}{c}\text { Androstenedione } \\
(\mathrm{nmol} / \mathrm{l})\end{array}$ & $\begin{array}{c}\text { Testosterone } \\
(\mathrm{nmol} / \mathrm{l})\end{array}$ & $\begin{array}{c}\text { Biologically } \\
\text { available } \\
\text { testosterone } \\
\text { (pmol/l) }\end{array}$ \\
\hline \multicolumn{6}{|l|}{ Fertile women } \\
\hline Follicular phase & $4.84-27.90$ & $2.63-11.00$ & $0.79-3.98$ & $0.10-1.53$ & $2.24-28.88$ \\
\hline Preovulation phase & $6.61-32.62$ & $2.49-11.24$ & $1.12-5.73$ & $0.21-1.39$ & $4.79-30.86$ \\
\hline Ovulation phase & $6.16-37.47$ & $2.52-11.29$ & $1.12-5.73$ & $0.28-2.02$ & $6.24-40.56$ \\
\hline Luteal phase & $6.30-32.34$ & $2.60-10.59$ & $0.54-4.93$ & $0.24-1.49$ & $0.71-27.78$ \\
\hline Postmenopausal women & $2.64-29.60$ & & $0.76-5.10$ & $0.05-1.59$ & \\
\hline \multicolumn{6}{|c|}{ Pregnant women - 37th week } \\
\hline Fetus-male & $2.50-12.87$ & & $0.85-8.17$ & $0.73-5.79$ & \\
\hline Fetus-female & $1.43-18.6$ & & $0.94-6.08$ & $0.99-5.07$ & \\
\hline
\end{tabular}

Reference ranges were calculated based on the 2.5th-97.5th percentiles of measured data. DHEA, testosterone and androstenedione were quantified using previously published LC-MS/MS method (Sosvorova et al. 2015, Vitku et al. 2016). DHEAS and SHBG (calculation of free testosterone) were measured using radioimmunoanalytic kits from Immunotech (Beckman Coulter Company, Marseille, France). Albumin (calculation of free testosterone) was determined using immunoanylytic kit from Roche (Basel, Switzerland). Biologically available testosterone was calculated according Vermeulen et al. (1999) http://www.issam.ch/freetesto.htm.

For calculating ranges in fertile women, 220 samples were analyzed from healthy women (average age 29.8 years, average BMI 24.56) that were not using any medications or hormonal contraceptives, had a regular menstrual cycle, and had no clinical signs of hyperandrogenemia. Dividing into four phases of the menstrual cycle was performed based on the day of the cycle and LH levels.

For calculating reference ranges in postmenopausal women, 97 samples were analyzed from healthy postmenopausal women (average age 58 years) that were not using any medications influencing steroidogenesis.

For calculating reference ranges at the 37th week of pregnancy, samples from 80 pregnant women were used (28 expecting boys and 52 expecting girls) with a physiological course of gravidity (average age 31.41 years, average weight gain during pregnancy $15.5 \mathrm{~kg}$ ) that were not using any medications.

All samples were taken in the morning hours between 7:30 and 9:30 AM.

Testosterone is the main androgen, with a daily production of 0.1-0.4 mg, but not the most effectual (this is dihydrotestosterone). In women, half of this amount is converted in the periphery from precursors, while the other half is divided equally between the adrenal glands and ovaries. Higher testosterone levels, or even better 
higher free testosterone or the FAI index, are key diagnostic information for doctors evaluating dermatological signs of hyperandrogenemia. However, in some women with hirsutism androgen levels have been found to be within reference ranges, so a biological marker may be more sensitive than a laboratory marker. Measuring testosterone is also important for diagnoses of androgen-producing tumors, where total testosterone levels can be 2-3x higher than reference levels. The level of androgenemia is also a useful marker for diagnostics and monitoring of androgenization disorders.

\section{Dihydrotestosterone (DHT)}

Dihydrotestosterone (DHT) is measured using direct immuoanalysis, which is affected by uncertainties arising from cross-reaction with testosterone. This is especially true at the low levels seen in women. Only a few laboratories offer DHT assays. More accurate results can be obtained using liquid chromatography, but this method is used rather for research purposes (Swerdloff et al. 2017).

DHT is metabolized from testosterone by an irreversible reduction catalyzed by the enzyme $5 \alpha$-reductase. This enzyme also affects the metabolism of progesterone, deoxycorticosterone and testosterone, and exists in three isoforms. DTH is the strongest androgen, with a $5 \mathrm{x}$ higher affinity to the androgen receptor than testosterone, resulting in androgen efficiency about double or triple that of testosterone in target tissues (Dušková and Pospíšilová 2011). Daily changes in DHT levels are not marked, though some studies have described decreases after awakening and in reaction to food intake (Racz et al. 2015). Unlike testosterone, changes during the menstrual cycle have not yet been described (Swerdloff et al. 2017), but this could be due to the analytical problems when analyzing minor changes. However, even assays using gas chromatography have not been able to demonstrate changes during the cycle or in the course of aging in low DHT levels in women (0.24-0.75 nmol/1) (Kannenberg et al. 2018).

The role of DHT in men has been well described, but its importance in women is unclear. Experimental models have shown a role in ovarian folliculogenesis (Murray et al. 1998, Rodrigues et al. 2015), but results from these models are not applicable to humans since steroidogenesis differs widely among species. Higher local levels of DHT could play a role in the development of androgenic alopecia in women who have normal blood levels. However, increased activities of any as well as all or none $5 \alpha$-reductase isoenzymes have all been described in women with androgenic alopecia, reflecting the heterogeneity of this disease and explaining the failure of many treatments (Sánchez et al. 2018). Many studies have attempted to find a role for DHT in hyperandrogenism, but the results were unfortunately ambiguous and DHT analysis is not useful in diagnosing hyperandrogenic states (Swerdloff et al. 2017).

\section{Methods for measuring androgens in the past and today}

\section{Individual methods for measuring and their limitations}

Measuring total testosterone levels in serum is the first choice when evaluating excessive androgen levels in women. Testosterone levels in women are about ten times lower than in men. This complicates their analysis, as they must achieve adequate sensitivity and reliability for measuring such low testosterone levels. Most European laboratories use immunoassays without prior extraction. This method allows very rapid analysis, but at the expense of low accuracy (Pugeat 2017). Higher accuracy can be obtained using radioimmunoassay (RIA) with extraction. However, only the use of extraction methods meets the recommendations for measuring testosterone. For improving the quality of results, liquid chromatography with tandem mass spectrometry (LC-MS/MS) should be considered, which provides the highest accuracy with low detection limits. This is especially useful for measuring the low testosterone levels in women. Instrumental analysis allows significantly improved results, but the institutional limitations (higher cost, the necessity for trained personnel, and technical limits (such as the influence of the matrix on analyses or interference by conjugates) complicate its routine use.

The first RIA of insulin published by Nobel Prize winners Yalow and Berson (1959) in 1977 opened the possibility of measuring other hormones. Odell et al. (1967) published the first RIA analysis of luteinizing hormone (LH), and Abraham (1969) described the first RIA analysis of a steroid hormone, estradiol. In the Czech Republic, Hampl et al. (1970) first published binding analysis of testosterone.

The first steroid RIA by Abraham (1969) for analyzing circulating estradiol levels included separation of estradiol from serum or plasma by extraction with an organic solvent and column chromatography before quantification with a radioactively-labeled marker. These 
two steps are important for the accuracy of the method. The organic solvent denatures proteins, including those which bind steroids as SHBG Column chromatography is used to separate estradiol from other steroids, mainly from its many metabolites that could interfere. This method was validated and was shown to be sensitive, specific, and precise (Stanczyk 2007).

RIA with purification (extraction and chromatography) was gradually adapted for measuring further steroids including androgens. These precise, sensitive, and specific methods have been used in many studies analyzing the relationships among steroids and in individual pathological states, and have become the foundation of diagnostic criteria. In addition to the clear benefits, however, the methods have some limitations. Among the main problems are the costs and time requirements, which make classical RIA practically inconvenient for routine clinical practice. For this reason, other newer methods have been developed. At the end of the 1970s, the first direct RIA was developed, which did not require the extraction and chromatographic steps. A further step forward was a change of reagents and the development of direct immunoanalytical methods with non-radioactive markers, which could be assessed on small-scale analyzers.

Direct immunoassays are simple, comfortable, rapid, and relatively inexpensive methods for measuring steroids, and thus advantageous for routine clinical practice. Unfortunately, however, they also have a number of disadvantages. The methods often suffer from insufficient specificity, and thus compared to classical RIA most values are overestimated. For constructing calibrations curves only standard solutions are often used, which differ from serum matrix and can thus markedly influence the results. With direct analysis some steroids (for instance testosterone and estradiol) may be insufficiently released from their binding to SHBG (lacking a purification step), and only an uncertain fraction is therefore measured. Non-conjugated steroids in the blood are largely bound to albumin, and some (with $17 \beta$-hydroxy groups) also to SHBG. The binding to albumin is weak, and the antibodies used during immunoassays possess by orders higher affinity, and so the binding to albumin is easily overcome. However, binding to SHBG is much stronger and for corrected measurements of total steroid levels it is necessary to break these bindings. In classical RIA this was accomplished by the organic solvent, which denatured SHBG. In direct immunoassay this can be achieved by a change in $\mathrm{pH}$ or by adding a substance that frees the steroid. The efficiency of this step is important for accuracy of the method (Stanczyk 2006). In light of the above-mentioned analytic difficulties, direct immunoanalyses have insufficient sensitivity for measuring some steroids, particularly when they have low serum concentrations (Stanzcyk 2006, Kane et al. 2007).

At the turn of the millennium several studies were published comparing individual methods used to measure testosterone in routine practice, documenting the problems with testosterone analyses especially at the low concentrations found in women. One of these studies compared ten commercially available methods for measuring testosterone (direct immunoassay without extraction), used most often in routine laboratories (Taieb et al. 2003). Herold and Fitzgerald (2003) summarized the results of method comparisons as follows: most currently used immunoanalyses missed their target by as much as $200-500 \%$, showing that guessing results would be a "better alternative" and could "provide less costly and quicker results of testosterone in women, without the need to take a blood sample".

A positive step forward in the new millennium was the move to chromatography with mass spectrometry, and this method became the gold standard. The basic principle of mass spectrometry was described more than a century ago, but its wider use was limited by the ability to measure only molecules of low molecular weight. A large step forward was made in the 1980s, when technical innovations widened the possible analyses. In the 1990s advances were introduced from the use of gas chromatography with mass spectrometry (GC-MS) to liquid chromatography with mass spectrometry (LC-MS), which began to be used in large laboratories (Grebe and Singh 2011). Commercial kits for LC-MS were produced, which allowed the method to be introduced to routine practice.

However, over time it was found that commercial kits for chromatography with mass spectrometry had their own problems that greatly decreased their accuracy. The analytical sets as designed do not have great advantages over immunoassay, and suffer from high costs, both for the test itself and for the personnel and technical equipment of the laboratory. Each laboratory must perform its own standardization and validation, though this should increase result accuracy. Simply transferring the measurement of testosterone to LC-MS does not by itself lead to more accurate results, and as discussed below, standardization of the method in each laboratory is necessary. 
A program for standardizing the measurement of testosterone

The problems with measuring testosterone led the Centers for Disease Control and Prevention (CDC) in the USA to initiate a program in 2007 for standardized measurement of serum testosterone (Vesper and Botelho 2010), which was finalized in the form of a specific method (Vesper et al. 2014). The concept and aim of standardized testosterone assays was inspired by the standardization of glycosylated hemoglobin (HbAlc) with the National Glycohemoglobin Standardization Program (NGSP) that began in 1993, since the variability in measuring glycosylated hemoglobin was at that time similar to the current situation with testosterone. The success of the NGSP was documented in 2002, when the majority of certified methods had an inter-laboratory $\mathrm{CV}$ lower than $5 \%$ and all certified methods measured HbAlc with an error within $0.8 \%$ of the values determined by the NGSP (Vesper and Botelho 2010).

Laboratories can participate in the program to standardize testosterone assays under a certification program, which can improve the quality of their testosterone assays. The cost of the "CDC Hormone Standardization Program (HoSt) - Testosterone Certified" program is $\$ 18,000$ plus fees (CDC 2013). As of April 2018, ten laboratories had currently certified assays and six laboratories had previously certified assays with no current certification (CDC 2018).

Comparisons of testosterone measurements from 142 accredited laboratories in the USA took place from 2012-2013. The participating laboratories used various immunoanalytical and chromatographic methods to measure testosterone. As a part of the study laboratories measured 4 serum samples with different testosterone concentrations, two from women and two from men. $73 \%$ of laboratories measured at least 3 samples correctly with a total error $\pm 25 \%$. In serum samples from female patients with a testosterone concentration of $15 \mathrm{ng} / \mathrm{dl}(0.5 \mathrm{nmol} / \mathrm{l})$, the results ranged from 8-36 ng/dl (0.3-1.3 nmol/1). This wide range of results demonstrated that low concentrations of testosterone remain a difficulty for laboratories. Serum from male patients were measured more accurately, but for a serum concentration of $402 \mathrm{ng} / \mathrm{dl}(14 \mathrm{nmol} / \mathrm{l})$, which according to recent recommendations is within the normal range for plasma, $15 \%$ of laboratories measured levels lower than $300 \mathrm{ng} / \mathrm{dl}$ (10.4 nmo/l), i.e. hypogonadal. This study showed that even chromatographic methods are not an automatic solution, but also verified that laboratories that underwent the certification program were able to produce quality results. Therefore, standardization of the methods should still be an ongoing goal (Cao et al. 2017).

\section{Recommendations and initiatives of the American Endocrine Society}

As a result of studies that evaluated and compared testosterone measurements from individual laboratories, the American Endocrine Society released a statement on the utility, limitations, and pitfalls of measuring testosterone (Rosner et al. 2007). This statement summarized the current status and provided recommendations for physicians and for the use of androgen examinations:

- It is necessary to know the type and quality of the method used, including reference ranges that must be created by each laboratory in cooperation with endocrinologists. In order to define reference ranges, it is necessary to define and characterize the parameters to be measured.

- For low concentrations of testosterone, direct measurements without extraction give inaccurate results and should not be used. Measurements with extraction and chromatography with either mass spectrometry or immunoassay give better results and are preferred.

- Most assays can distinguish testosterone levels in normal and hypogonadal men. However, to diagnose hypogonadism in men it is recommended to always perform more than one examination. Sampling must be done in the morning hours.

- When using a quality assay method and welldefined reference ranges, total serum testosterone levels taken in the follicular phase of the menstrual cycle are recommended as an initial test for suspected androgenproducing tumors in women.

- When using a quality assay method for measuring testosterone and SHBG with well-defined reference ranges, calculated free testosterone is a clinically sensitive marker of hyperandrogenemia in women and can be used in combination with clinical symptoms for diagnosis and monitoring these patients.

- If not a part of hypophyseal insufficiency, measurements of testosterone used for evaluating sexual dysfunction or fatigue in adult women are not supported by any published data, and are therefore very strictly not recommended.

- In children, reference ranges must be adjusted for sex, age, and stage of sexual maturation, and must be specific for the given assay method if a universal standard 
is not available.

- Measuring total testosterone in children has limited value. Evaluating excess androgen, virilization, intersexual disorders or maturation with signs of the opposite sex are indicated only for girls. There are only a few reasons to measure testosterone in boys, among them for evaluating gonadal failure, disorders of sexual maturation or puberty, and checking the response to treatments.

In 2010 the Endocrine Society organized an interdisciplinary conference with the aim to improve the quality of testosterone measurement, attended by representatives from the American Association for Clinical Chemistry, American Association of Clinical Endocrinologists, and other specialized organizations (Androgen Excess/PCOS Society, American Society for Bone and Mineral Research, American Society for Reproductive Medicine, American Urological Association, Association of Public Health Laboratories, Laboratory Corporation of America, North American Menopause Society and Pediatric Endocrine Society). The outcome of the conference was a recommendation to improve the current possibilities for measuring testosterone at the system level and planning individual steps for the future (Rosner et al. 2010).

\section{The problems with reference ranges}

Most previous studies dealing with androgen levels, their physiological roles, and relationships to various pathologies used RIA with purification, while current studies use mostly chromatography with mass spectrometry. Recommendations for the diagnosis of hyperandrogenism with a specific cut-off based on these studies are very difficult to carry over to clinical practice, since routine laboratories generally use other methodologies. Blindly using reference norms and recommendations across all analytic methods can lead to the risk of incorrect interpretations of laboratory androgen assays, and is one of the most fundamental mistakes.

The terms "normal ranges" and "normal values" have been replaced by the term "reference ranges", in order to avoid having a specific definition of "normal". The reference range (value) is the interval of the $2.5^{\text {th }}-97.5^{\text {th }}$ percentile of concentration values of a given analyte found in a defined population (determined in a population of reference individuals, i.e. individuals with a defined state of health) (Racek et al. 2006).

The problems with reference ranges have two aspects. The first arises from the difference between the methods used and the second from physiological changes to individual steroids. Each laboratory must determine their own reference ranges for evaluating steroid levels in serum, plasma, saliva or urine. Only by using their own references ranges their results could be correctly interpreted, since these can significantly differ between individual laboratories and various methodologies even when performed correctly. Defining reference ranges for androgens in women is a significant problem particularly in gynecological endocrinology. The main problems are that the concentration of circulating testosterone is relatively low, relatively small changes can be negatively manifested on somatic parameters, and mainly because concentrations are affected by numerous influences depending on temporal factors. Its levels change physiologically with age (over the course of years), reproductive function status (months to years), menstrual cycle (weeks), but also with seasonal and daily biorhythms. All these aspects cannot be reflected in reference ranges, and thus it is necessary to respect the day of the cycle and time of day that are defined as standard for taking samples. For assessing physiological steroid changes, it is thus necessary to use different ranges not just for women and men, but also for age groups and for women the phase of their menstrual cycle.

\section{Conjugated and non-conjugated androgens}

Conjugated/non-conjugated steroids should not be confused with the terms free and bound steroids, which are used for describing binding to proteins (see the next chapter).

Steroid hormones are secreted from production glands mainly non-conjugated, with the exception of DHEAS, which is secreted directly from adrenal as sulphate. Their binding to sulfuric, glucuronic or other acids leads to the production of so-called conjugates, which used to be thought of as the final degradation products of steroid hormones. These conjugates are soluble in water, enabling their excretion in the urine. Today we know that even these conjugated steroids often have functional roles, which are sometimes completely different than their non-conjugated counterparts, such as the case of dehydroepiandrosterone (DHEA) and its sulfate (DHEAS) in ion channels in the brain.

\section{Free and bound androgens}

In the blood androgens are mostly bound to proteins, and only the small free fraction is considered to 
be biologically active. Non-conjugated steroids are mainly bound to albumin, and those that have a 17-hydroxy group are also bound to SHBG. Most DHEA and androstenedione are bound to albumin. In contrast, only $30-40 \%$ of total testosterone is bound to albumin and $65 \%$ is bound to SHBG.

Testosterone circulates in the blood in three forms. The most important is free testosterone, which is a biologically active form ready for use by target organs and tissues. This makes up only about $2-3 \%$ of the total testosterone. The next testosterone fraction is bound to albumin. Albumin has a low binding affinity to the testosterone molecule and can easily be removed, and so this fraction is considered to be biologically available for other tissues. The sum of free and albumin-bound testosterone is therefore sometimes termed biologically available testosterone. The largest fraction of testosterone is bound to SHBG, which is a glycoprotein insuring the transport of testosterone through the circulatory system. SHBG has a high binding affinity to testosterone and this fraction is biologically non-active, serving as a hormone reserve. Total testosterone is thus the sum of free testosterone, testosterone bound to albumin, and testosterone bound to SHBG.

In women, determining the fraction of free or biologically available testosterone is important because SHBG levels are significantly influenced not only by thyroid gland function, obesity, and insulinemia, but also by concentrations of circulating estrogens. Signs of hyperandrogenemia in women with testosterone levels within reference ranges can often be caused by a high amount of active testosterone.

In the Czech Republic, analytically measured free testosterone is not commonly available and is generally accompanied by various errors. The equations used to calculate free testosterone have a number of inadequacies, but still provide important information, particularly in cases where there are significant changes to concentrations of binding proteins. Measuring free testosterone is analytically difficult. Concentrations are most accurately quantified using equilibrium dialysis. Another possibility is to obtain the fraction not bound to SHBG by precipitating SHBG-bound testosterone with ammonium sulfate. Both of these methods are accurate, but for routine use are impractical, since they are technically quite difficult, and as such are mostly just used in research. In routine practice, free testosterone is commonly measured using methods analogous to those used to analyze free thyroid gland hormones. These assays use a testosterone analog with low affinity to SHBG and albumin, which in theory allows just the free testosterone fraction to be measured. However, in principle it is clear that this is not as accurate as equilibrium dialysis.

To estimate free testosterone in clinical practice, therefore, either direct measurements of free testosterone or non-direct calculation methods may be used. The results obtained from direct measurements are difficult to compare to those from calculations. On the other hand, directly measured free testosterone may supplement information gained from calculations. Equations may seem like the simplest way to obtain values of free testosterone, but they have limits arising from the measurement accuracy for individual analytes and correctly determining reference ranges for free testosterone. This second limitation is of course also applicable when measuring free testosterone.

Calculations of the active fraction of total testosterone most commonly use: 1) The free androgen index (FAI), which is the ratio of total testosterone to SHBG: FAI $(\%)=$ (total testosterone/SHBG) $\times 100$. 2) An estimate of biologically available testosterone, which is calculated using the level of albumin in addition. Calculated biologically available testosterone is then the sum of free testosterone and albumin-bound testosterone.

An accurate way to calculate free or biologically available testosterone was made possible by the equations of Vermeulen. Theses mathematical algorithms use measurements of the total testosterone, SHBG and albumin concentrations. They include the association constants for interaction of testosterone with SHBG and albumin and give results comparable to measured values of free testosterone obtained by equilibrium dialysis. The Vermeulen equation is thus useful for estimating free testosterone in women with the exception of free testosterone in pregnant patients (Vermeulen et al. 1999).

A detailed description of calculations of Vermeulen's "free" and "biologically available" testosterone can be found at the following site, where these can be easily obtained after entering values of albumin, testosterone, and SHBG: http://www.issam.ch/freetesto.htm.

\section{Measurements in different bodily fluids}

Measuring androgens in serum or plasma

Both serum and plasma can be used to measure 
steroid hormones. It is generally accepted that serum is more appropriate due to the possible interference of small precipitates that occur when freezing and thawing plasma. This is mainly important when performing measurements in small plasma volumes $(0.05 \mathrm{ml})$. Most laboratories therefore recommend assays be done with serum (Stanczyk 2006).

One limitation of using serum or plasma is that the results reflect only the current concentration of a given steroid, so it must be remembered that there may be fluctuations during the day, menstrual cycle or due to other influences. This must be kept in mind when planning sampling and interpreting results.

In acute cases when it is necessary to measure androgens from plasma instead of serum or vice versa, this is possible but the results from different fluids cannot be compared. It must also be accepted that these are just estimated measurements. Laboratories also generally report normal ranges determined for only one of these bodily fluids.

In serum and plasma androgens are present in non-conjugated forms (DHEA, androstenedione, testosterone) and conjugated forms like sulfates or glucuronides (DHEAS).

\section{Measuring androgens in urine}

Measurements performed in urine collected over $24 \mathrm{~h}$ are advantageous because they provide information on the secretion of a given steroid over this whole period and eliminate short-term concentration fluctuations. Unfortunately, key limitations of such assays are the difficulty of precisely collecting urine and in cases of kidney diseases, which can alter the excretory ability.

As opposed to in serum/plasma, in the urine steroids mainly occur in conjugated form, mostly as glucuronides. The body transforms lipophilic non-conjugated steroids to water-soluble conjugates that can be excreted in the urine. For this reason only conjugated steroids can be measured in urine (Stanczyk 2006).

Measurements of androgens in urine were first performed using a colorimetric assay of 17-ketosteroids or 17-oxosteroids, which are present in the urine mostly in conjugated form and which are mostly metabolites of adrenal androgen precursors. 17-ketosteroids are therefore mainly a reflection of the function of the adrenal glands, not the ovaries.

In an effort to eliminate the problems arising from testosterone concentration fluctuations in serum/plasma under certain circumstances, a new method has been developed for measuring testosterone in urine. The analysis of testosterone in urine involves several critical steps (hydrolysis of glucuronides, purification of non-conjugated steroids using chromatography, and quantification using RIA). Because of the difficulties, this method is unfortunately not applicable for routine practice (Stanczyk 2006).

\section{Measuring androgens in saliva}

Sampling of saliva has the advantage of being non-invasive, simple, and providing an opportunity for repeated multiple samplings during a given time period. However, interpretations of results are complicated by the contamination of exogenous steroids from the diet (for instance eggs), the possible contamination of the saliva by blood, and further by changes in concentrations of steroids caused by steroid metabolizing enzymes present in the salivary glands. Concentrations in saliva therefore differ to various degrees from serum/plasma levels. As opposed to urine, in saliva most steroids are present in non-conjugated form.

Testosterone in saliva is present at about 2-3\% of serum concentrations. Because concentrations of testosterone in saliva are roughly similar to concentrations of free testosterone in the plasma, it has been hypothesized that saliva testosterone reflects concentrations of free testosterone. There is no evidence for this theory, and it is not based on fact, since binding proteins also occur in the saliva. Differences in testosterone concentrations in saliva depend on enzymes in the salivary glands, which convert androstenedione to testosterone and then metabolize it (Stanczyk 2006). In contrast to testosterone, a good correlation has been found for saliva and plasma/serum levels of DHEA and androstenedione.

It must be again repeated that in women the low concentrations of testosterone in serum/plasma make analytic assays problematic. Measurements in saliva are even more difficult, and its use in clinical practice questionable.

\section{Additional examinations for the differential diagnosis of hyperandrogenemia}

If after overcoming all analytical difficulties higher androgen levels are found in female patients, as part of a differential diagnosis it becomes necessary to distinguish between the various states associated with 
hyperandrogenemia. First it is necessary to distinguish the relatively rare causes of increased androgens from ovarian or adrenal tumors that produce androgens. Then it is necessary to distinguish non-classical forms of adrenal hyperplasia, for which measurements of 17a-hydroxyprogesterone and a confirmatory Synacthen test can be performed. Cushing's syndrome can be diagnosed using both clinical symptoms and laboratory tests: the dexamethasone suppression test, higher levels of cortisol in urine, and diurnal variations of the $\mathrm{d}$ cortisol. Overproduction of androgens is usually caused by the hypothalamic-pituitary-ovarian axis, which leads to our final difficulty, defining polycystic ovarian syndrome, which is undergoing a constant dynamic development. And last-but-not-least, hyperandrogenism can occur in patients with clinical signs of androgenization without any laboratory evidence of increased androgen levels.

\section{Conclusion}

The diagnostics of hyperandrogenemia in women is accompanied by a range of difficulties, which can be divided into several types, each of which is important for the interpretation of results. The fundamentals are a correct indication for performing measurements, the choice of analytes to be measured, and ruling out the use of medications that influence steroid production. Next, the correct procedures in the preanalytic phase should be observed considering the physiological changes in steroids in women, which is important not just for androgens but for all steroids in general. Sampling should be performed between 8 and 10 in the morning (if the patient awakes before $7 \mathrm{am}$, due to the circadian rhythm and cortisol response to awakening), in the follicular phase of the menstrual cycle, i.e. from day 1-5 (due to the influence of the menstrual cycle), after fasting (due to the influence of food), while relaxed (due to the influence of stress), and keeping the age of the patient in mind. Other important factors are knowledge of the limits of the methods the laboratory uses, in what sample measurements are performed, and of the requirements for correct reference ranges. Following these recommendations allows for a correct interpretation of results without putting a strain on patients, performing unnecessary examinations, or causing stress due to mysterious diagnoses, while ensuring that serious diseases associated with hyperandrogenemia are not overlooked.

\section{Conflict of Interest}

There is no conflict of interest.

\section{Acknowledgements}

The study was supported by a project of the Ministry of Health of the Czech Republic - RVA („,Endokrinologický ústav - EÚ00023761“) and grant 17-30528 A of the MZ ČR AZV.

\section{References}

ABRAHAM GE: Solid-phase radioimmunoassay of estradiol-17 beta. J Clin Endocrinol Metab 29: 866-870, 1969. ADAMCOVÁ K, KOLÁTOROVÁ L, JANDÍKOVÁ H, CHLUPÁČOVÁ T, ŠMÍDOVÁ M, PAŘÍZEK A, STÁRKA L, DUŠKOVÁ M: Changes to fetal steroidogenesis caused by maternal smoking. Physiol Res 66 (Suppl 3): S375-S386, 2017.

CAO ZT, BOTELHO JC, REJ R, VESPER H: Accuracy-based proficiency testing for testosterone measurements with immunoassays and liquid chromatography-mass spectrometry. Clin Chim Acta 469: 31-36, 2017.

CDC Laboratory/Manufacturer Hormone Standardization (HoSt) Program, Standardization of Serum Total Testosterone measurements (2013) https://www.cdc.gov/labstandards/pdf/hs/Testosterone_Protocol.pdf

CDC Hormone Standardization Program (HoSt) - Testosterone Certified Procedures (UPDATED 03/2018) https://www.cdc.gov/labstandards/pdf/hs/CDC_Certified_Testosterone_Procedures-508.pdf

DUŠKOVÁ M, POSPÍŚILOVÁ H: The role of non-aromatizable testosterone metabolite in metabolic pathways. Physiol Res 60: 253-261, 2011.

DUŠKOVÁ M, ŠIMU゚NKOVÁ K, HILL M, VELÍKOVÁ M, KUBÁTOVÁ J, KANCHEVA L, KAZIHNITKOVÁ H, HRUŠKOVIČOVÁ H, POSPÍŠILOVÁ H, RÁCZ B, SALÁTOVÁ M, CIRMANOVÁ V, KRÁLÍKOVÁ E, STÁRKA L, PAŘÍZEK A: Chronic cigarette smoking alters circulating sex hormones and neuroactive steroids in premenopausal women. Physiol Res 61: 97-111, 2012.

GREBE SK, SINGH RJ: LC-MS/MS in the clinical laboratory - where to from here? Clin Biochem Rev 32: 5-31, 2011. 
HAMPL R, RABOCH J, STÁRKA L: Determination of testosterone using the protein-binding method and its level in male plasma (in Czech). Čas Lék Čes 109: 616-619, 1970.

HEROLD DA, FITZGERALD RL: Immunoassays for testosterone in women: better than a guess? Clin Chem 49: 1250-1251, 2003.

JANDÍKOVÁ H, DUŠKOVÁ M, ŠIMU゚NKOVÁ K, RÁCZ B, HILL M, POSPÍŠILOVÁ H, KMEŤOVÁ A, KRÁLÍKOVÁ E, VONDRA K, STÁRKA L: How smoking cessation influence hormonal levels in postmenopausal women? Prague Med Rep 115: 60-66, 2014.

KANE J, MIDDLE J, CAWOOD M: Measurement of serum testosterone in women; what should we do? Ann Clin Biochem 44: 5-15, 2007.

KANNENBERG F, FOBKER M, SCHULTE E, PIERŚCIŃSKI G, KELSCH R, ZITZMANN M, NOFER JR, SCHÜRING AN: The simultaneous measurement of serum testosterone and $5 \alpha$-dihydrotestosterone by gas chromatography-mass spectrometry (GC-MS). Clin Chim Acta 476: 15-24, 2018.

MURRAY AA, GOSDEN RG, ALLISON V, SPEARS N: Effect of androgens on the development of mouse follicles growing in vitro. J Reprod Fertil 113: 27-33, 1998.

ODELL WD, ROSS GT, RAYFORD PL: Radioimmunoassay for luteinizing hormone in human plasma or serum: physiological studies. J Clin Invest 46: 248-255, 1967.

PUGEAT M: Hyperandrogenetic states: pitfalls in diagnostic approach. Endocrine Abstracts 49: S18.1, 2017.

RACEK J, EISELT J, FRIEDECKÝ B, HOLEČEK V, NEKULOVÁ M, PITTROVÁ H, RUŠAVÝ Z, SENFT V, ŠAVLOVÁ M, TĚŠÍNSKÝ P, VERNER M: Clinical Biochemistry (in Czech). Second edition. Galén, Prague, 2006, $329 \mathrm{p}$.

RÁCZ B, DUŠKOVÁ M, VONDRA K, ŠRÁMKOVÁ M, STÁRKA L: Daily profiles of steroid hormones and their metabolites related to food intake. Physiol Res 64 (Suppl 2): S219-S226, 2015.

RODRIGUES JK, NAVARRO PA, ZELINSKI MB, STOUFFER RL, XU J: Direct actions of androgens on the survival, growth and secretion of steroids and anti-Müllerian hormone by individual macaque follicles during three-dimensional culture. Hum Reprod 30: 664-674, 2015.

ROSNER W, AUCHUS RJ, AZZIZ R, SLUSS PM, RAFF H: Position statement: utility, limitations, and pitfalls in measuring testosterone: an Endocrine Society position statement. J Clin Endocrinol Metab 92: 405-413, 2007.

ROSNER W, VESPER H; ENDOCRINE SOCIETY; AMERICAN ASSOCIATION FOR CLINICAL CHEMISTRY; AMERICAN ASSOCIATION OF CLINICAL ENDOCRINOLOGISTS; ANDROGEN EXCESS/PCOS SOCIETY; AMERICAN SOCIETY FOR BONE AND MINERAL RESEARCH; AMERICAN SOCIETY FOR REPRODUCTIVE MEDICINE; AMERICAN UROLOGICAL ASSOCIATION; ASSOCIATION OF PUBLIC HEALTH LABORATORIES; ENDOCRINE SOCIETY; LABORATORY CORPORATION OF AMERICA; NORTH AMERICAN MENOPAUSE SOCIETY; PEDIATRIC ENDOCRINE SOCIETY: Toward excellence in testosterone testing: a consensus statement. J Clin Endocrinol Metab 95: 4542-4548, 2010.

SÁNCHEZ P, SERRANO-FALCÓN C, TORRES JM, SERRANO S, ORTEGA E: 5 $\alpha$-reductase isozymes and aromatase mRNA levels in plucked hair from young women with female pattern hair loss. Arch Dermatol Res 310: 77-83, 2018.

SOSVOROVA L, VITKU J, CHLUPACOVA T, MOHAPL M, HAMPL R: Determination of seven selected neuro- and immunomodulatory steroids in human cerebrospinal fluid and plasma. Steroids 98: 1-8, 2015.

STANCZYK FZ: Measurement of androgens in women. Semin Reprod Med 24: 78-85, 2006.

STANCZYK FZ: Androgen measurements. Methods, interpretation, and limitations. In: Androgen Excess Disorders in Women. AZZIZ R, NESTLER JE, DEWAILLY D (eds), Human Press Inc, Totowa, NJ, 2007, pp 63-72.

STÁRKA L, RÁCZ B, ŠRÁMKOVÁ M, HILL M, DUŠKOVÁ M: Daily profiles of dehydroepiandrosterone and its hydroxylated metabolites with respect to food intake. Prague Med Rep 116: 40-48, 2015.

SWERDLOFF RS, DUDLEY RE, PAGE ST, WANG C, SALAMEH WA: Dihydrotestosterone: biochemistry, physiology, and clinical implications of elevated blood levels. Endocr Rev 38: 220-254, 2017.

ŠRÁMKOVÁ M, DUŠKOVÁ M, VÍTKŮ J, VČELÁK J, MATUCHA P, BRADNOVÁ O, DE CORDEIRO J, STÁRKA L: Levels of adipokines and some steroids during the menstrual cycle. Physiol Res 64 (Suppl 2): S147-S154, 2015. 
TAIEB J, MATHIAN B, MILLOT F, PATRICOT MC, MATHIEU E, QUEYREL N, LACROIX I, SOMMADELPERO C, BOUDOU P: Testosterone measured by 10 immunoassays and by isotope-dilution gas chromatography-mass spectrometry in sera from 116 men, women, and children. Clin Chem 49: 1381-1395, 2003.

VESPER HW, BOTELHO JC: Standardization of testosterone measurements in humans. J Steroid Biochem Mol Biol 121: 513-519, 2010.

VESPER HW, BOTELHO JC, WANG Y: Challenges and improvements in testosterone and estradiol testing. Asian $J$ Androl 16: 178-184, 2014.

VERMEULEN A, VERDOCK L, KAUFMAN JM: A critical evaluation of simple methods for the estimation of free testosterone in serum. J Clin Endocrinol Metab 84: 3666-3672, 1999.

VITKU J, HERACEK J, SOSVOROVA L, HAMPL R, CHLUPACOVA T, HILL M, SOBOTKA V, BICIKOVA M, STARKA L: Associations of bisphenol A and polychlorinated biphenyls with spermatogenesis and steroidogenesis in two biological fluids from men attending an infertility clinic. Environ Int 89-90: 166-173, 2016.

YALOW RS, BERSON SA: Assay of plasma insulin in human subjects by immunological methods. Nature 184: 1648, 1959. 